\title{
Auditorías de seguridad vial como políticas para mejorar la seguridad del transporte en la provincia de Chimborazo- Ecuador
}

\author{
Ángel Paredes García \\ Docente Principal, Escuela de Civil, Facultad de Ingeniería, Universidad Nacional de \\ Chimborazo, Km 1 1⁄2 vía a Guano, Riobamba, Ecuador.
}

\section{RESUMEN}

La provincia de Chimborazo localizada en el centro del país se encuentra atravesada por una de las principales carreteras denominadas Troncal de la Sierra, la misma que conecta Colombia, Ecuador y Perú. Una auditoría de seguridad vial es un análisis formal que pretende garantizar que un camino, en nuestro caso existente, cumpla con criterios óptimos de seguridad. Dichas auditorías deben ser llevadas a cabo por un equipo de profesionales, cuyos miembros sean independientes del proyecto de la vía. En los últimos dos años la Universidad Nacional de Chimborazo, con sus estudiantes de pregrado, ha realizado Auditorías de Seguridad Vial en las principales vías que ingresan a la provincia de Chimborazo con el fin de reducir los accidentes en dichas carreteras. Gracias a los resultados obtenidos en base a una lista de chequeos realizados en cada vía, se pudo establecer que pese al alto porcentaje de seguridad presente en las vías, existen todavía puntos conflictivos, los mismos que deben ser intervenidos inmediatamente para disminuir el índice de accidentabilidad en nuestras carreteras. Las soluciones más comunes resultado de las auditorías realizadas son: instalación correcta de la señalización, diseño de taludes, iluminación y, de ser necesario, rediseño de la vía. Además se pudo establecer parámetros para determinar las políticas o futuros planes de acción con el fin de mejorar la seguridad en las vías y por ende la seguridad en el transporte.

Palabras Clave: Seguridad vial, políticas para mejorar la seguridad del transporte, auditoría de seguridad vial.

\section{INTRODUCCIÓN}

El propósito de la investigación es elaborar las políticas o acciones a tomarse luego de realizar una Auditoría de Seguridad Vial (ASV), con el fin de reducir los accidentes en las principales vías que ingresan a la provincia de Chimborazo, basándose en una lista de chequeos realizadas a cada vía, identificando y evaluando datos tanto de señalización, iluminación, obras de arte, obras de drenaje, derecho de vía, diseño geométrico, que cumplan con los parámetros exigidos en la seguridad vial, estos fueron comparados con los parámetros del Nivel de Servicio y categorías de acción del Índice de Calidad del Pavimento (PCI) de una vía, para luego proponer políticas que se deberán implementar según los 
resultados obtenidos.

Las ASV presentan a priori varios beneficios: Reducir la probabilidad que se produzcan accidentes en la red de carreteras, reducir la gravedad de los accidentes que inevitablemente se producen, adquirir una mayor conciencia de seguridad vial en los responsables del diseño, reducir el costo de medidas paliativas en la fase de explotación y reducir el costo económico y social de los accidentes.

"Dinamarca, Australia, Nueva Zelanda y Reino Unido son algunos de los países que han introducido las ASV en los procesos de gestión de la seguridad vial. Otros países, como Estados Unidos, Canadá o Alemania han desarrollado procedimientos para la realización de ASV, incluidas listas de chequeo, e incluso han realizado auditorías piloto en sus carreteras, con vistas a la implantación generalizada del proceso. La perspectiva británica considera que las listas de chequeo deben constituir una guía para la realización de ASV, no un listado que se rellene sistemáticamente. Sin embargo, la revisión de la normativa publicada en 2003 para la realización de ASV en carreteras en servicio incluye una versión más detallada de las listas de chequeo. (Díaz, J.)".

\section{MARCO TEÓRICO}

\subsection{Definición de Auditoría de Seguridad Vial}

Una Auditoría en Seguridad Vial se entiende como un proceso formal de revisión de un proyecto, de una carretera nueva antes de que ésta sea abierta al tránsito, de los trabajos de conservación y mantenimiento, de la operación y funcionamiento, o de cualquier otro proyecto que afecte las condiciones de circulación de un camino. En el curso de una auditoría en seguridad, un experto o equipo de expertos calificados e independientes analizan el nivel de seguridad, poniendo de manifiesto todos y cada uno de los riesgos potenciales; el objetivo principal es identificar y atender dichos riesgos desde las primeras etapas del proyecto, en el entendido de que las medidas correctivas que se apliquen resultan más caras una vez construido el camino. (Mayoral, Contreras, y otros. 2001)

\subsection{Métodos para llevar a cabo una Auditoría}

Existen tres métodos por los cuales se puede llevar a cabo una ASV:

- Ejecutada por un auditor o equipo de auditores, especialistas e independientes del equipo encargado del diseño.

- Realizada por el personal del mismo departamento encargado del diseño, pero personas diferentes a los encargados del proyecto original.

- Llevada a cabo por el mismo equipo encargado del proyecto.

El más recomendado es el primero ya que es el único que garantiza la independencia que debe existir entre el proyectista y el auditor, tal y como se recomienda desde la definición 
misma de una auditoría. El éxito de una ASV, depende de la verdad y compromiso de todas las partes; en una auditoría se identifican y señalan las deficiencias existentes desde el punto de vista de la seguridad de los usuarios, y esto puede ser visto como una amenaza para los encargados del diseño o la ejecución de un proyecto. (Mayoral, Contreras, y otros)

\subsection{Plan Nacional de Seguridad Vial}

Dentro de los pilares de acción del Plan Nacional de Seguridad Vial (PNSV) el pilar 2: Vías de tránsito y movilidad más seguras, tiene como objetivo: “Aumentar la seguridad de la Red Vial Nacional en todas sus fases de gestión". Y dentro de sus políticas tenemos:

- Establecer la obligatoriedad de la aplicación de parámetros de seguridad definidos por MTOP, en el desarrollo de proyectos integrales de infraestructura vial.

- Garantizar el cumplimiento de normas y reglamentos en las infraestructuras viales existentes y su respectivo mantenimiento.

- Fortalecer los conocimientos y las capacidades técnicas de las entidades públicas y privadas en la gestión de la infraestructura vial.

- Asegurar la inclusión de las necesidades de todos los usuarios de las vías en la planificación y construcción de la infraestructura vial. (Agencia Nacional de Tránsito 2013)

\section{MATERIALES Y MÉTODOS}

\subsection{Tipo de Investigación}

La investigación es descriptiva ya que las auditorías de seguridad vial se realizaron en las principales vías que ingresan a la provincia de Chimborazo como son: Riobamba - Ambato; Balbanera - Pallatanga; Riobamba - Chunchi y la Penipe - empalme Ambato-Baños, las mismas que fueron divididas en tramos de $2,5 \mathrm{Km}$ para recopilar información existente y poder comparar con los parámetros de seguridad e identificar la ubicación de los sitios más conflictivos.

Para la identificación de los parámetros que no cumplen con la seguridad vial, fue necesario recorrer las vías tantas veces se necesitó, tanto en el día como en la noche y analizar las mismas desde el punto de vista del conductor como del peatón o usuario de la vía, marcando con una X si cumple o no cumple los Ítems que nos presenta la lista de chequeo, para luego calificar con 1 el casillero marcado con $\mathrm{X}$ y 0 el que no está.

\subsection{Metodología de la Investigación}

La metodología de investigación se basa en una investigación de campo e investigación documental, para lo cual se necesitó realizar las auditorías de seguridad vial donde se utilizó la Lista de Chequeo General ASV a Vías Existentes del Compendio de Manuales de Seguridad Vial del FONSAT. Las mismas que cuentan con los siguientes Ítems: 
- Alineamiento y sección transversal: donde se analiza

- Visibilidad; distancia de visibilidad

- Diseño de velocidad

- Límite de velocidad/velocidad dividida por zonas

- Adelantamientos

- Legibilidad para conductores

- Anchos

- Bermas o espaldones

- Pendiente Transversal

- Drenaje

- Pistas Auxiliares

- Canalizaciones

- Bermas

- Señalización vertical y demarcación

- Intersecciones

- Localización

- Visibilidad; distancia de visibilidad

- Regulación y delineación

- Diseño

- Tipo de material

- Señalización Vertical e Iluminación

- Iluminación

- Aspectos generales de las señales verticales

- Legibilidad de las señales verticales

- Soporte de la señalización vertical

- Demarcación y Delineación

- Línea central, línea de borde y línea de pistas

- Delineadores y retro reflectantes

- Advertencias y delineación de curvas

- Barreras de contención

- Barreas de contención

- Terminaciones

- Peatones

- Alcances generales (peatones)

- Transporte público

- Puentes y Alcantarillas

- Características de diseño

- Barreras de contención y delineación

- Pavimentos

- Defectos en el pavimento

- Estancamiento 
- Varios

- Trabajos temporales

- Problemas de encandilamiento

- Actividades de borde de la vía

- Animales

- Contención de taludes

Una vez identificados y calificados los Ítems se procedió a sumar todos los tramos para tener un resultado final donde nos indican el total de tramos seguros e inseguros que tiene cada vía, para luego llevarlo a porcentaje de valoración.

\begin{tabular}{|l|c|c|c|}
\hline \multicolumn{4}{|c|}{ EJEMPLO DE VALORIZACIÓN } \\
\hline $\begin{array}{c}\text { Alineamiento y sección } \\
\text { transversal }\end{array}$ & \multicolumn{2}{|c|}{ CUMPLE } & ANÁLISIS \\
\hline \multicolumn{2}{|c|}{ SI } & NO & \\
\hline Drenaje & & & \\
\hline $\begin{array}{l}\text { Los canales del drenaje al borde de } \\
\text { la vía y las paredes de las } \\
\text { alcantarillas pueden ser atravesados } \\
\text { en forma segura por los vehículos? }\end{array}$ & & X & \\
\hline
\end{tabular}

\begin{tabular}{|l|c|c|c|}
\hline \multicolumn{4}{|c|}{ EJEMPLO DE VALORIZACIÓN } \\
\hline $\begin{array}{c}\text { Alineamiento y sección } \\
\text { transversal }\end{array}$ & \multicolumn{2}{|c|}{ CUMPLE } & ANÁLISIS \\
\hline \multicolumn{2}{|c|}{ SI } & NO & \\
\hline Drenaje & & & \\
\hline $\begin{array}{l}\text { Los canales del drenaje al borde de } \\
\text { la vía y las paredes de las }\end{array}$ & 0 & 1 & INSEGURO \\
$\begin{array}{l}\text { alcantarillas pueden ser atravesados } \\
\text { en forma segura por los vehículos? }\end{array}$ & 0 & & \\
\hline Total: & 0 & 1 & \\
\hline
\end{tabular}

Tabla 1 - Ejemplo de Valorización de las listas de chequeo

\section{RESULTADOS Y DISCUSIÓN}

\subsection{Resultados de las ASV realizado en las vías.}

Realizadas las ASV de las cuatro principales vías que ingresan a la provincia de Chimborazo, cuyos resultados se presenta en la figura 1, se identifica primero que la vía que tiene más observaciones con porcentaje de inseguridad es la vía Riobamba-Chunchi, que además es la vía que tiene mayor longitud y que la vía más segura es la vía Cahuají empalme AmbatoBaños que es la de menor longitud. Con esto tampoco se puede decir que la inseguridad de las vías es directamente proporcionales a la longitud de las mismas. 


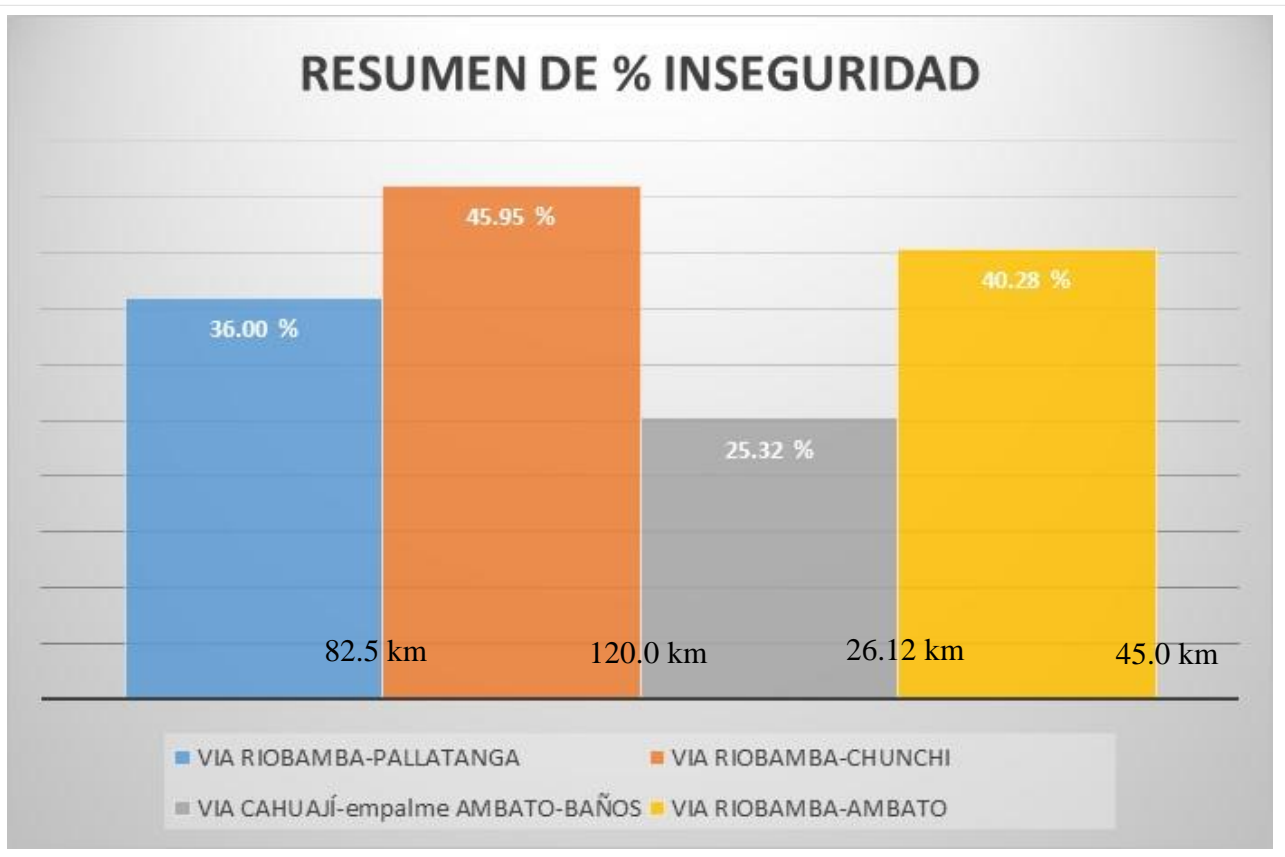

Fig. 1 - Resumen del porcentaje de inseguridad de las vías en estudio.

\subsection{Procesamiento y Análisis}

Una vez obtenidos los resultados con las técnicas usadas estos se interpretaron de manera lógica para poder dar soluciones, identificando cualquier deficiencia de seguridad vial, siendo los auditores los encargados con su conocimiento y experiencia de mejorar el análisis. Los resultados del análisis según los ítems analizados se presentan en la siguiente figura 2.

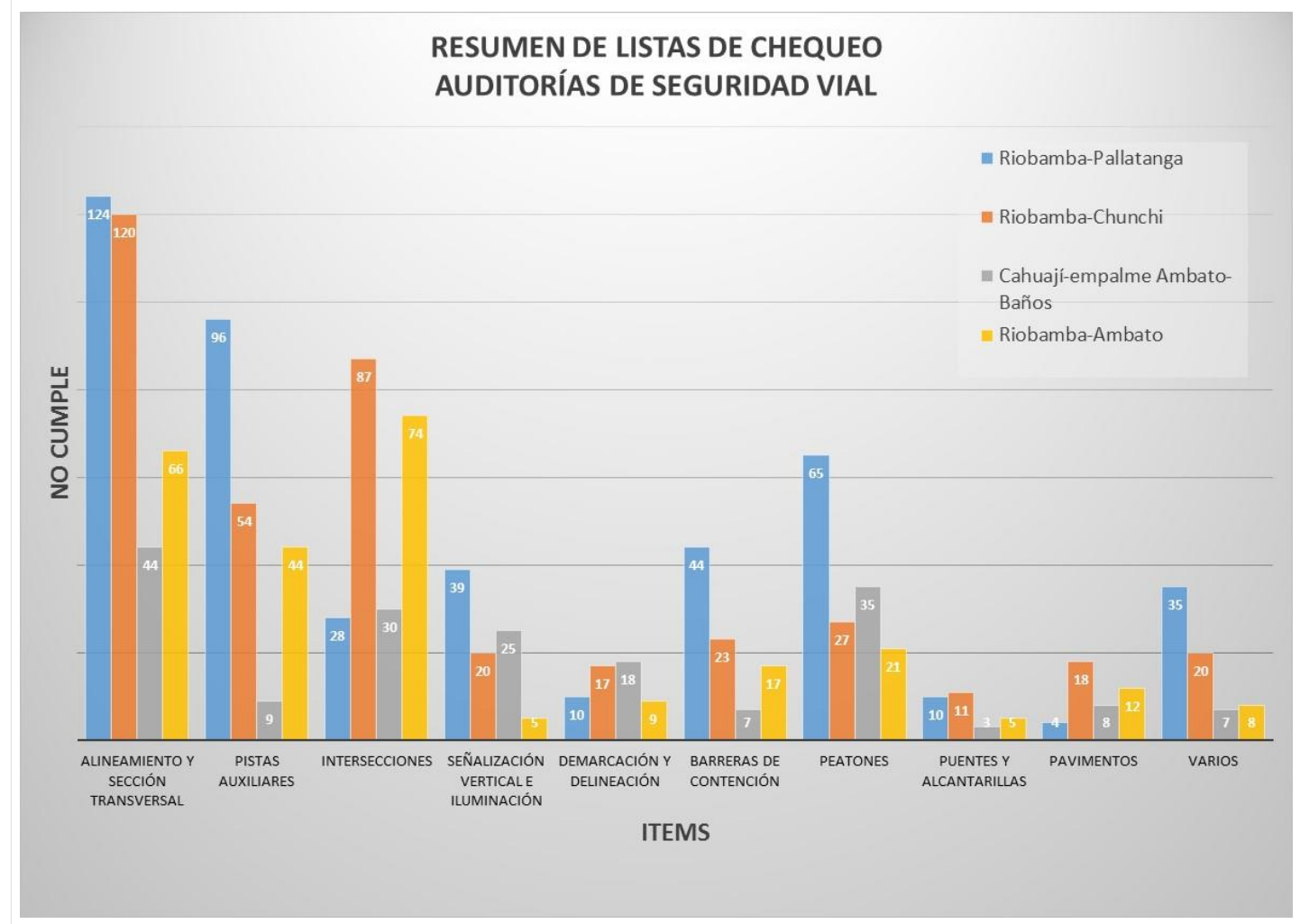

Fig. 2 - Resumen de las listas de chequeo de las ASV. 


\subsection{Discusión.}

Al comparar el porcentaje de inseguridad de las vías se observa que se puede hablar de un rango o parámetro donde se puede calificar la seguridad de la vía desde excelente hasta fallido, pasando por muy bueno, bueno, regular, malo y muy malo. Calificación que permite recomendar las acciones o políticas a tomarse según los resultados obtenidos y que pueden ser comparados con las recomendaciones que se hacen con el Nivel de Servicio, tabla 2.

\begin{tabular}{|c|c|c|l|l|}
\hline \multicolumn{2}{|c|}{ NIVEL DE SER VICIO Y CATEGORÍA DE ACCIÓN DEL ICP(PCI) } \\
\hline 5 & Muy bueno & Mantenimiento rutinario & $\begin{array}{l}\text { Pavión ICP } \\
\text { percibidionto por lon usuarios es satisfactorio. Ocasionalmente se presentan } \\
\text { pequeños daños que no afectan significativamente la circulación y pueden } \\
\text { ser evitados o corregidos en el mantenimiento rutinario. }\end{array}$ \\
\hline 4 & Bueno & $\begin{array}{c}\text { Mantenimiento rutinario y } \\
\text { recurrente }\end{array}$ & $\begin{array}{l}\text { Pavimento en condición buena, la circulación es cómoda. Se presentan } \\
\text { daños localizados en etapa de iniciación. }\end{array}$ \\
\hline 3 & Regular & $\begin{array}{c}\text { Refuerzo - mantenimiento } \\
\text { rutinario }\end{array}$ & $\begin{array}{l}\text { Pavimento en estado regular, en donde la circulación deja de ser cómoda. } \\
\text { Se presentan daños de manera constantemente en etapas avanzadas. }\end{array}$ \\
\hline 2 & Malo & Rehabilitación & $\begin{array}{l}\text { Pavimento en condición mala, la circulación es muy incomoda. Se } \\
\text { presentan daños en etapas muy desarrolladas. }\end{array}$ \\
\hline 1 & Muy malo & Reconstrucción & $\begin{array}{l}\text { Pavimento en condición muy mala, la vía se vuelve intransitable. Los } \\
\text { deterioros están muy desarrollados y son irreversibles. El pavimento está } \\
\text { totalmente degradado. }\end{array}$ \\
\hline
\end{tabular}

Tabla 2 - Niveles de Servicio y Categorías de Acción del ICP.

Podemos observar además que los alineamientos, secciones transversales y las intersecciones, son los ítems que más calificación de porcentaje de inseguridad se encuentra y los ítems de puentes y alcantarillas son los que menos observaciones de inseguridad fueron encontradas. Los mismos que nos dan una idea de clasificación y calificación de los ítems para según esto tomar las acciones o políticas a desarrollarse.

Sin embargo no podemos decir que el diseño geométrico de las vías son los deficientes ya que en la mayoría de los tramos de las vías estudiadas, estas cumplen con los parámetros mínimos de diseño tanto horizontal como vertical, lo que no se toma en cuenta en el diseño es el concepto de zona despejada a lo largo de la vía, esto más por problemas económicos ya que el incluir en el estudio y en la construcción este concepto, encarecería la obra.

Las vías en estudio clasifican con una calificación de buena a regular, calificación que tomamos de la tabla 3. Donde las políticas a desarrollar serán:

- Corregir puntos críticos de señalización vertical, iluminación y varios, y

- Rehabilitar la seguridad del peatón y barreras de contención en su orden. 


\begin{tabular}{|l|c|c|}
\hline \multicolumn{1}{|c|}{ POLITICAS O ACCIÓN A TOMARSE } & CALIFICACIÓN & \% INSEGURO \\
\hline $\begin{array}{l}\text { Realizar Mantenimiento rutinario de Puentes y } \\
\text { Alcantarillas }\end{array}$ & Exelente & $0-5$ \\
\hline $\begin{array}{l}\text { Realizar Mantenimiento periódico de Demarcación y } \\
\text { Delineación }\end{array}$ & Muy Bueno & $5-20$ \\
\hline $\begin{array}{l}\text { Corregir puntos críticos de Señalización Vertical, } \\
\text { Iluminación y Varios }\end{array}$ & Bueno & $20-35$ \\
\hline $\begin{array}{l}\text { Rehabilitar seguridad de Peatones y Barreras de } \\
\text { contención }\end{array}$ & Regular & $35-50$ \\
\hline Rehabilitación de tramos más afectados & Malo & $50-65$ \\
\hline Rehabilitación total & Muy Malo & $65-80$ \\
\hline Reconstrucción Total & Fallido & $80-100$ \\
\hline
\end{tabular}

Tabla 3 - Políticas a tomarse según el porcentaje de inseguridad.

\section{AGRADECIMIENTOS}

Un agradecimiento especial a los estudiantes de pre grado que realizaron las Auditorías de Seguridad Vial en cada una de las vías en estudio. Y al Ing. Jorge Núñez docente de la Facultad de Ingeniería por su valioso aporte para la culminación de este trabajo.

\section{REFERENCIAS}

- AGENCIA NACIONAL DE TRÁNSITO DEL ECUADOR. Plan Nacional de Seguridad Vial. Semana de Seguridad Vial (2013)

- BRAVO, F.X. y PEÑAFIEL, A.K. (2013) Evaluación de la Seguridad Vial en la carretera de primer orden Riobamba-Ambato.

- CASTILlO, G.A. y DONOSO, C.E. (2014) Evaluación de Seguridad Vial en la carretera de primer orden Riobamba-Chunchi.

- DIAZ, J. Auditorías de Seguridad Vial. Experiencia en Europa.

- FONSAT. (2011) Compendio de Manuales de Seguridad Vial I-II-III

- GOMEZ, R.L. y GOMEZ, P.E. (2014) Auditoría en Seguridad Vial de la carretera de primer orden Riobamba-Pallatanga.

- MAYORAL, CONTRERAS y otros. (2001) Auditorías en Seguridad Carretera. Procedimientos y Prácticas. Publicación Técnica No. 183. Sanfandila, Qro. 2001

- PACHECO, L.F. y PACHECO, A.F. (2015) Evaluación de la Seguridad Vial en la carretera Cahuají empalme vía Ambato-Baños, ubicada en las provincias de Chimborazo y Tungurahua. 